CARNETS DE Carnets de géographes

GÉOGRAPHES.

$1 \mid 2010$

Varia

\title{
Lire le lieu pour dire la ville
}

Florentin : une mise en perspective d'un quartier de Tel Aviv dans la mondialisation (2005-2009)

\section{Caroline Rozenholc}

\section{(2) OpenEdition}

\section{Journals}

Édition électronique

URL : http://journals.openedition.org/cdg/2291

DOI : $10.4000 /$ cdg. 2291

ISSN : 2107-7266

Éditeur

UMR 245 - CESSMA

\section{Référence électronique}

Caroline Rozenholc, «Lire le lieu pour dire la ville », Carnets de géographes [En ligne], 1 | 2010, mis en ligne le 01 octobre 2010, consulté le 24 septembre 2020. URL : http://journals.openedition.org/cdg/ 2291 ; DOI : https://doi.org/10.4000/cdg.2291

Ce document a été généré automatiquement le 24 septembre 2020

\section{(c) $(1)$}

La revue Carnets de géographes est mise à disposition selon les termes de la Licence Creative Commons Attribution - Pas d'Utilisation Commerciale - Pas de Modification 4.0 International. 


\section{Lire le lieu pour dire la ville}

Florentin : une mise en perspective d'un quartier de Tel Aviv dans la mondialisation (2005-2009)

\section{Caroline Rozenholc}

\section{Génie du lieu et spatialisation des identités : une problématique remobilisée}

1 La mondialisation est souvent évoquée comme une ère de déstabilisation, d'accélération et de désaffiliation territoriale. Elle semble en effet faire se multiplier ce que M. Augé (1992) avait appelé des «non-lieux », des lieux dépourvus de toute valeur identificatoire, sans âme et sans portée sociale. Toutefois, alors même que l'équation nous liant à certains lieux est remise en mouvement par la part qu'y occupent les mobilités, les modalités de l'appropriation spatiale demeurent une problématique géographique centrale.

2 À travers ce travail de thèse, il s'agit donc de réinterroger le sens du lieu dans le contexte urbain et c'est le quartier de Florentin, à Tel Aviv, qui constitue l'espace pour observer les transformations du lieu mondialisé et saisir les réarrangements induits par la mondialisation de la ville et du pays. Un peu à la marge d'une ville au dynamisme saisissant, de cette « bulle » d'insouciance et de prospérité dans une réalité tourmentée, le quartier de Florentin fonctionne dans cette recherche comme un prisme à travers lequel déchiffrer le quotidien d'une société israélienne en pleine transformation.

Pour saisir les implications locales de dynamiques mondiales et le jeu d'échelles entre local et global dans un lieu défini, chacune des trois parties de la thèse - «Explorations géographiques d'un quartier historique ", «Florentin dans la mondialisation » et «Entre exotisme et nostalgie. Globalisation d'un quartier 'authentique'" - vient décliner une facette du quartier de Florentin, tout à la fois singulier dans le paysage de la ville et particulièrement représentatif de l'histoire politique et des mouvements d'immigration qui ont fait Tel Aviv. 


\section{Identités et identifications : enjeux d'une réflexion située dans un quartier de Tel Aviv}

4 Créé dans les années 1920 à l'articulation de Tel Aviv et de Jaffa, à un moment crucial de l'histoire de la Palestine mandataire, le quartier de Florentin est, dès la création de l'État d'Israël, rapidement abandonné des services publics. Espace de première installation des populations immigrées et poumon industriel et économique de la ville, Florentin se dépeuple et se dégrade toujours plus jusqu'au milieu des années 1990.

5 Il devient, à ce moment-là, un espace d'hospitalité réciproque, voire de mobilisation civile et culturelle, pour la jeunesse bohème et les travailleurs immigrés, venus d'Europe, d'Asie et d'Afrique. Il rassemble alors l'ensemble des strates que constitue la population résidant en Israël : citoyens israéliens juifs - et parmi eux de nombreux «nouveaux immigrés »- et arabes, des Palestiniens de Cisjordanie, des résidents « temporaires » tels que les travailleurs migrants ou les requérants d'asile.

6 Aujourd'hui, en pleine fièvre immobilière, Florentin est investi par une population en quête cette fois d'exotisme et d'authenticité. Et ce quartier d'environ 6000 habitants devient le lieu où déjouer, avec une certaine nostalgie, les évolutions sociétales très rapides du pays. Dans une agglomération partagée en trois zones administratives (Tel Aviv Nord, Centre et Sud) où s'oppose dans des fonctionnalités complémentaires le Nord et le Sud de la ville, Florentin est demeuré à l'écart des grands projets immobiliers et de la transformation du tissu urbain à l'œuvre ailleurs dans la ville.

7 Si Florentin est de longue date un quartier dont les conditions socio-économiques sont des plus précaires, sa géohistoire et l'aura d'effervescence qui accompagne les dernières rénovations le démarquent également des quartiers adjacents. Dans des processus détaillés tout au long de la thèse, une des "qualités » particulières de Florentin est de donner à voir que le contenu des espaces ne s'abolit pas au cours du processus social mais qu'il en subsiste toujours 'quelque chose' qui se fond en une ambiance spécifique. L'atmosphère de Florentin décrite par différents interlocuteurs, faite de convivialité et de marginalité, de tolérance, de pauvreté et d'embourgeoisement, de superposition de modes de vie somme toute et de pratiques urbaines - industries, logements, commerces, loisirs -, vient d'ailleurs témoigner des réarrangements que produit la mondialisation dans les espaces les plus fluctuants des grandes villes. Dans l'exemple de Florentin, ces fluctuations mettent à jour des lieux jusque-là oubliés, enfouis, puis dévoilés par les transformations de la ville.

\section{Méthode pour une géographie sensible}

8 Dans ce travail, les propos recueillis au cours de près de 80 entretiens sur cinq ans (entre 2005 et 2008) avec des habitants du quartier, des représentants des services de planification de la municipalité et des institutions publiques ou privées actives à Florentin et dans les quartiers Sud, constituent l'une des principales sources d'information. Les documents d'archive permettent également de mettre la géohistoire du quartier en regard de la place que celui-ci occupe dans la trame de la ville, mais faisant le lien entre coprésence et traces, l'exploration du lieu par la rue est l'une des lignes directrices de ce travail. 
9 La « rue » permet de saisir les différentes temporalités, signes et acteurs dans leurs configurations infiniment variables et leurs expressions identitaires quotidiennes. Elle est, par excellence, le lieu de la coprésence et de la confrontation, des aménagements et des tensions. En Israël, et en particulier dans des espaces interstitiels tels que Florentin, c'est un lieu d'observations particulièrement instructif où s'enregistrent présences et traces, affiches et graffitis. Relevés sur les murs du quartier, leur dimension revendicative, mais aussi ludique, est évidente. L'observation et la production de nombreux clichés photographiques permettent alors de rapporter la manière dont les usagers agencent des fragments de sens à travers leurs pratiques.

\section{Florentin : un terrain fertile de la pensée}

10 Focaliser notre recherche sur un quartier de Tel Aviv indique la priorité donnée aux « réarrangements » des lieux produits par la mondialisation sur les questions politiques les plus évidentes dans un espace qui attise les passions identitaires bien au-delà de ses propres frontières. C'est alors de manière détournée, par le caractère situé des phénomènes observés à Florentin, par les manifestations quotidiennes et banales de l'ancrage local et de l'identification au lieu, que l'on approche des questions nationales ou régionales cruciales telles que le débat sur l'identité israélienne et la présence sur le territoire national. Opérant la conjonction d'époques et de passages successifs, ce lieu entre-deux contient en effet en germe tout un faisceau de problématiques et de questionnements qui autorise de monter en généralisation. Le quartier de Florentin témoigne de la démultiplication du lieu, ouvert mais contenu dans des limites flexibles, et peut être, à ce titre, entrepris comme caisse de résonance et de diffraction des registres. Révélant la tension entre proche et lointain, de l'exotisme chez soi et des notions d'authenticité et de nostalgie qui lui sont associées, ce travail circule entre des registres de sens différents, en s'attachant à lire le lieu pour dire la ville contemporaine et celle qui se profile pour demain. Ce travail creuse ainsi le sillon de la réflexion sur le rapport au lieu dans une temporalité mondialisée, d'une territorialité dans son sens le plus large de lien entre individus et entités spatiales du quotidien.

\section{Fiche informative}

Lien vers la thèse

http://tel.archives-ouvertes.fr/tel-00496355/fr/

\section{Discipline \\ Géographie}

\section{Directeur}

W. Berthomière et E. Ma Mung

\section{Université}

Université de Poitiers, laboratoire Migrinter (UMR 6588).

Membres du jury de thèse, soutenue le 21 mai 2010.

- M. William Berthomière, Chargé de recherche au CNRS, Migrinter, Poitiers

- Mme Chantal Bordes-Benayoun, Directrice de recherche au CNRS, Lisst-centre

d'anthropologie sociale, Toulouse 
- M. Yankel Fijalkow, Professeur des Universités, École d'Architecture Paris Val de Seine, rapporteur

- Mme Adriana Kemp, Professeur, Département de sociologie et d'anthropologie, Université de Tel Aviv

- M. Emmanuel Ma Mung, Directeur de recherche au CNRS, Migrinter, Poitiers

- M. Jean-François Staszak, Professeur, Université de Genève, rapporteur

Situation professionnelle actuelle

post-doctorante au laboratoire Migrinter (UMR 6588)

Contact de l'auteur

caroline.rozenholc[at]univ-poitiers.fr

INDEX

Thèmes: Carnets de soutenances 